Hodgson. T. A. (1989) Cost of illness studies: no aid to decision making? Comments on the second opinion by Shiell et al. Health Policy, 11, 57-60.

KNAPP, M. (1997) Costs of schizophrenia. British Journal of Psychiatry. 171, 509-518.

RoviRA, J. (1995) Economic analysis and pharmaceutical policy. Anaesthesia. 50 (suppl.), 49-51.

Shiell. A.. Gerard, K. \& DONALDSON. C. (1987) Cost of illness studies: an aid to decision making? Health Policy, 8, 317-323.

NEIL CRAIG, Health Economist, Department of Public Health, University of Glasgow, 2 Lilybank Gardens, Glasgow G12 8RZ; and CAMERON STARK, Consultant in Public Health Medicine, Highland Health Board. Beechwood Park. Inverness IV2 3HG

\section{Members of a community mental health team}

Sir: Lucas concludes that his study of community mental health team members' activity should inform service planning (Psychiatric Bulletin. September 1997, 21, 547-549). There are however limitations to a study that only counts the number of staff-patient face-to-face contacts and classifies each as 'assessment', 'ongoing' or 'group' and cross tabulates these by discipline.

Lucas does not describe the data collection method in any detail and the reliability of the data appears not to have been established (e.g. through case-note or diary audit). Lucas makes no comment on the remarkably low contact rate of 1.4 patients per full clinical day. In our experience of collecting staff activity data, mental health professionals' compliance with even very clear data collection protocols is variable. Such data collection requires continuous and careful attention to detail if meaningful conclusions are to be drawn.

The paper's most serious problems lie in the interpretation of the data. Lucas' claim that "the CMHNs [Community mental health nurses] had the most face-to-face contacts followed by the consultant ... Psychologists and occupational therapists ... social workers then junior doctors" is only true if one ignores the number of staff from each profession. For example there are 5 whole time equivalent whole (WTE) CMHNs and only 1.4 social workers (WTE). When we calculated and ranked the number of contacts (WTE) per week from Lucas' data a very different picture emerged. The consultants see the most patients (18.5) followed by psychologists (12.1), social workers (11.8), junior psychiatrists (8.2) and occupational therapists (6.2). CMHNs have the least number of contacts (5.2).

Lucas' assertion that a team geared towards assessments ought to maximise psychiatric staffing (although possibly true) is not supported by his data. In one of his study teams the halftime consultant sees $40 \%$ of those being assessed. In the other the fifth-time consultant sees none. Decisions about team staffing must be based on firmer evidence than the current practice in one of two study teams.

Lucas did not measure duration of contact. His statement, therefore, that "CMHNs spent more than $50 \%$ of their time with [patients with severe mental illness]... while social workers spent less than $20 \% "$ can only be speculation.

There is no doubt that team composition is a vital component in community mental health services and Lucas is right to assert that there is currently little information to guide service planners in this respect. However, it is important that the research evidence that forms the basis of service planning is of high quality.

MATTHEW FIANDER, Research Fellow, ROB BALE, Lecturer in Community Psychiatry, and TOM BURNS, Professor of Community Psychiatry. St George's Hospital Medical School, Section of Community Psychiatry, Division of General Psychiatry. Jenner Wing. Cranmer Terrace, London SW17 ORE

Author's reply: I am grateful to Fiander et al for highlighting some of the limitations of this kind of study, both in terms of collecting staff activity data and interpreting it. They suggest that staff compliance with activity data collection may be poor. This arises from their experience, but it is difficult to comment on this when it is just that. For the teams studied, the activity data collected formed part of the contracting process with the purchasers (and samples were validated by them). The data were distributed on the trust network and were thus a means of communication, and it was used for case-load monitoring within the teams, so there were considerable pressures for professionals to comply.

Perhaps the most substantial point they raise does need clarifying: all the figures do already allow for the number of posts in each profession. Clearly it would be nonsensical to draw conclusions about professional roles by comparing the work of one part-time social worker with that of three full-time community psychiatric nurses.

Despite the limitations mentioned in the article and by Fiander et al, the study does begin to address the question of who should be in a community mental health team, and should form a basis with which to compare further, and perhaps more refined, results in this area.

BEN LUCAS, Specialist Registrar, Joint Homelessness Team. 8 King Street, London WC2E $8 \mathrm{HN}$ 